\title{
TME: Aplicativo M-Learning para o Estudo de Conceitos Matemáticos com Ênfase no ENEM
}

\author{
Hannderson Faria Arantes, Rodrigo Duarte Seabra \\ Instituto de Matemática e Computação - Universidade Federal de Itajubá (UNIFEI) \\ Caixa Postal 50 - 37.500-903 - Itajubá - MG - Brasil \\ hannderson.arantes@gmail.com,rodrigo@unifei.edu.br
}

\begin{abstract}
The ENEM (National Examination of Secondary Education) is an assessment developed by the Ministry of Education and aims to measure the domain of competences and skills of students who are completing high school. Currently, the test is widely used as a tool of access to the majority of public institutions of the country. Thus, the preparation of students is essential for obtaining good results during the competition for a place at higher education. This research presents the development of an application for the Android platform in order to assist the preparation of students for the ENEM in the context of the mathematics discipline. The main results showed that the tool is presented as a good alternative to be explored in the classroom.
\end{abstract}

Resumo. O ENEM (Exame Nacional do Ensino Médio) é uma avaliação elaborada pelo Ministério da Educação e tem como finalidade mensurar o domínio de competências e habilidades de estudantes que estão concluindo o Ensino Médio. Atualmente, o exame é amplamente utilizado como instrumento de acesso pela maioria das instituições públicas do país. Assim, a preparação dos estudantes é essencial para a obtenção de bons resultados durante a disputa por uma vaga no ensino superior. Esta pesquisa apresenta o desenvolvimento de um aplicativo para a plataforma Android visando auxiliar a preparação de estudantes para o ENEM, no contexto da disciplina de matemática. Os principais resultados mostraram que a ferramenta se apresenta como uma boa alternativa a ser explorada em sala de aula.

\section{Introdução}

Atualmente, vivencia-se uma árdua concorrência quando se trata de ingressar em uma instituição de ensino superior no Brasil. Por conta disso, faz-se necessária uma preparação intensa do estudante para que esteja apto a conquistar sua vaga em uma universidade.

Com o objetivo de avaliar estudantes no final do Ensino Médio, em 1998, foi criado o Exame Nacional do Ensino Médio (ENEM). Na atualidade, o exame é o principal meio de entrada para que estudantes ingressem em uma universidade brasileira. Segundo dados do Ministério da Educação (MEC 2015), o número de instituições que aderiram ao ENEM já ultrapassa 120 unidades.

Aliado a esse cenário, é cada vez mais perceptível que novos paradigmas vêm sendo aplicados no processo de ensino-aprendizagem, visando melhorar a compreensão dos assuntos estudados dentro e fora da sala de aula. Em relação a esse aspecto, aplicativos educacionais gratuitos estão à disposição de docentes e discentes por meio da internet, contribuindo para um ambiente de aprendizado mais diversificado e interessante.

Do ponto de vista de Marçal, Andrade e Rios (2005), com a ampla disseminação 
na utilização de dispositivos móveis, notou-se que esta tecnologia poderia ser empregada na educação como parte de um modelo de aprendizado integrado. $\mathrm{O}$ uso de dispositivos móveis na educação criou um novo conceito denominado mobile learning ou apenas $m$ learning. Já em 2002, Nyiri (2002) afirma que o paradigma m-learning surge da intenção de aproveitar a disponibilidade de dispositivos móveis, considerando as necessidades específicas de educação e treinamento. De modo complementar a essa premissa, Rheingold (2007) ressalta que a ubiquidade dos dispositivos móveis conduzirá o $\mathrm{m}$ learning como um meio importante para fornecer educação e informação. O poder ubíquo dos dispositivos móveis para se aprender em qualquer lugar e a qualquer momento tem conquistado cada vez mais adeptos que buscam não só complementar o ensino, mas realizar estudos nos mais diversos locais.

McGreal (2005) afirma que a aprendizagem concebida por meio de m-learning terá um impacto significativo na aprendizagem tradicional. Para este autor, num futuro próximo, esse tipo de aprendizagem se tornará parte habitual da educação de todos e complementará o contexto de aprendizagem da sociedade, levando este paradigma a ganhar pertinência e visibilidade.

A partir do cenário exposto, esta pesquisa tem como objetivo geral apresentar o desenvolvimento de um aplicativo que, possivelmente, privilegie o entendimento e o treinamento de conceitos matemáticos por meio de um aplicativo mobile gratuito disponível na plataforma Android. Espera-se, portanto, que o aplicativo educacional proposto auxilie os estudantes em sua preparação para a avaliação de matemática do ENEM, abordando conteúdos relacionados ao ensino desta disciplina. Adicionalmente, o trabalho ainda se propõe a investigar, sob a ótica discente, os potenciais benefícios apontados em decorrência do uso do aplicativo proposto no apoio ao ensino da matemática. Como principais contribuições do estudo destacam-se: (i) contribuir com a disseminação da ideia do uso de aplicativos mobile gratuitos, para que futuramente haja aumento de sua utilização no âmbito educacional; (ii) avaliar qualitativamente a opinião dos estudantes envolvidos no estudo, verificando se o aplicativo desenvolvido nesta oportunidade atendeu as expectativas esperadas, bem como seu grau de aceitação e contribuição no processo de aprendizagem.

Nesse sentido, a seção 2 discute a importância do ENEM no cenário educacional, o uso de aplicativos m-learning no ensino, bem como alguns trabalhos correlatos. A seção 3 discorre sobre o aplicativo desenvolvido neste estudo e o método empregado em sua avaliação. A seção 4 apresenta a análise dos dados coletados na pesquisa experimental realizada e, finalmente, a seção 5 apresenta as conclusões do trabalho.

\section{Fundamentação Teórica}

O ENEM, criado pelo Instituto Nacional de Pesquisas Educacionais Anísio Teixeira (INEP), do Ministério da Educação (MEC), tem como finalidade avaliar a qualidade do Ensino Médio no país, bem como possibilitar que estudantes ingressem no Ensino Superior. A avaliação é composta por uma redação e provas objetivas que avaliam as seguintes áreas de conhecimento: Linguagens, Códigos e suas Tecnologias, Ciências Humanas e suas Tecnologias, Ciências da Natureza e suas Tecnologias, e Matemática e suas Tecnologias (MEC 2015).

Desde a sua criação, o ENEM veio a constituir uma área de interesse de estudo tanto no âmbito político quanto no educacional, como pode ser visto nos trabalhos de 
Lopes e López (2010), com um estudo sobre a performatividade nas políticas de currículo no caso do ENEM, e de Castro e Tiezzi (2004), com uma pesquisa sobre a reforma do Ensino Médio e a implantação do ENEM no Brasil. Para Silva e Ribas (2009), o ENEM se difere de outros exames propostos pelo MEC, pois, além de centrarse em avaliar o desempenho do estudante por competências e habilidades, privilegia a interdisciplinaridade e a contextualização.

De acordo com Castro e Tiezzi (2004), o que está presente na concepção do ENEM é a importância de uma educação com conteúdos mais ricos, voltada para o desenvolvimento da capacidade cognitiva e de raciocínio dos estudantes. A formação não está mais pautada na memorização maciça de informações e fatos, mas sim no desenvolvimento de estruturas mentais que permitem ao jovem e ao adulto enfrentar problemas novos, usando as tradicionais teorias científicas.

Como uma das áreas do conhecimento abrangidas pelo ENEM, segundo Ponte et al (2007), a matemática é uma das ciências mais antigas e, igualmente, uma das mais tradicionais disciplinas escolares, tendo sempre ocupado um lugar relevante no currículo. Para Cazorla (2012), diversos indivíduos afirmam possuir aversão a essa disciplina e, para a maioria, isso acontece por lembranças de aprendizagens escolares. Uma possível explicação para isso se deve à falta de percepção das possíveis aplicações sobre o conhecimento estudado, bem como sua utilidade para a vida desses indivíduos. Ademais, de acordo com Carvalho (2009) e Ponte e Souza (2010), o fato de ainda, atualmente, as aulas de matemática em nível de primeiro, segundo e terceiro graus serem realizadas de forma expositiva, impede o desenvolvimento cognitivo do estudante. $\mathrm{O}$ aprendiz passa a realizar exercícios de aplicação, que nada mais são do que uma repetição da aplicação de um modelo de solução apresentado pelo docente.

Em oposição a essa prática, Marçal, Andrade e Rios (2005) defendem que os conceitos de m-learning favorecem um aprendizado dinâmico. Esta prática beneficia tanto trabalhadores como estudantes, que enfrentam um longo deslocamento até seu trabalho ou instituição de ensino, devido à dinamicidade de sua rotina ao envolver viagens ou translados para diferentes locais. A preocupação é fornecer um ambiente de aprendizado que proporcione a informação mais atualizada possível, bem como o suporte tanto para a aprendizagem formal como para a informal, fomentando meios para o desenvolvimento de métodos inovadores de ensino utilizando recursos da computação e da mobilidade. Desta forma, o m-learning surge como uma importante ferramenta alternativa de ensino, aprendizagem e treinamento à distância.

\subsection{Trabalhos Correlatos}

Batista, Behar e Passerino (2010), com o objetivo de promover uma classificação de aplicativos e objetos de aprendizagem sobre dispositivos móveis direcionados à matemática, realizaram uma análise criteriosa a respeito de alguns softwares comerciais e gratuitos. Foi constatado que a popularização dos dispositivos móveis é um aspecto positivo em termos educacionais, uma vez que favorece o alcance de um grande número de indivíduos, sem requerer deslocamentos físicos. A pesquisa também destacou que há necessidade de inovações no processo de ensino-aprendizagem no contexto da matemática, pois ainda são poucas as iniciativas que consideram o uso de dispositivos móveis como ferramenta de apoio.

No trabalho de Neto e Fonseca (2013), com o intuito de estimular o aprendizado da matemática utilizando jogos educativos para dispositivos móveis, foi desenvolvido um 
jogo, para a plataforma Android, baseado na obra literária de Malba Tahan: "O homem que calculava". Visando comprovar a efetividade de sua proposta, os resultados apontaram que a maioria dos estudantes aprovou o apoio da mobilidade no uso de jogos educativos digitais, devido à flexibilidade de uso independente do local.

Visando a apresentar uma elicitação dos principais requisitos do desenvolvimento de aplicações m-learning, sobretudo as destinadas ao ensino de matemática, Marçal et al (2010) definiram uma base para a criação de um guia prático para este tipo de aplicação. $\mathrm{Na}$ ocasião, foram desenvolvidos três aplicativos no âmbito educacional: M-Queops, MPrisma e M-Pitagoras. Os resultados da pesquisa apontaram que o emprego de dispositivos móveis se apresenta como uma interessante ferramenta de auxílio ao ensino e à aprendizagem. Além disso, foi constatado que o ensino-aprendizagem de conceitos matemáticos por meio de m-learning apresenta algumas particularidades que exigem desde a adaptação de conteúdos às pequenas telas até a adoção de métodos pedagógicos específicos.

$\mathrm{Na}$ pesquisa realizada por Batista, Behar e Passerino (2011), foi proposto um modelo pedagógico, denominado m-LearnMat, visando a orientar atividades de $m$ learning em matemática com foco no ensino superior. O modelo foi aplicado em turmas de Cálculo I de uma Instituição Federal de Ensino utilizando o celular como dispositivo móvel. Foi constatado que o modelo pedagógico proposto orientou a proposta da disciplina, que foi fortemente apoiada pelas tecnologias digitais.

O estudo realizado por Sena, Oliveira e Carvalho (2014) teve como objetivo apresentar um mapeamento sistemático da literatura sobre aplicações m-learning utilizadas no ensino de matemática, com conteúdo e recursos diferenciados e aplicados em diferentes níveis de ensino (Fundamental, Médio e Superior). Os resultados apontaram a importância de se analisar o impacto dos aplicativos m-learning, pois eles podem influenciar a motivação e o desempenho dos estudantes. Ademais, foi constatado que há necessidade de se promover inovações no processo de ensino-aprendizagem no contexto de matemática. Finalmente, os autores também destacaram que ainda são poucas as iniciativas que consideram o uso de dispositivos móveis como ferramenta de apoio.

A partir das pesquisas arroladas, é fato que estudos envolvendo o uso de aplicativos móveis têm despertado o interesse de pesquisadores no desenvolvimento de projetos diferenciados que facilitem o ensino e a aprendizagem de matemática. $\mathrm{Na}$ mesma direção dos estudos apresentados, o fator motivador envolvido nesta oportunidade também consiste em proporcionar apoio aos estudantes que, muitas vezes, demonstram ou relatam algum tipo de dificuldade na aprendizagem de matemática. Deste modo, o presente trabalho se apresenta como mais uma alternativa frente a esse desafio, abrangendo uma nova oportunidade para o treinamento de conceitos matemáticos como forma de preparação para o ENEM.

\section{Método}

\subsection{A Ferramenta TME}

O TME (Treinamento de Matemática para o ENEM) consiste em uma aplicação Android com a finalidade de fornecer apoio ao processo de treinamento para o ENEM na disciplina de matemática. A seleção da plataforma foi realizada de forma a abranger a maior quantidade possível de usuários. Para o desenvolvimento da aplicação, foi utilizada 
V Congresso Brasileiro de Informática na Educação (CBIE 2016)

Anais do XXVII Simpósio Brasileiro de Informática na Educação (SBIE 2016)

a IDE Android Studio, concebida pelo Google, que consiste em um ambiente para a criação de aplicações para a plataforma Android. O desenvolvimento ocorreu utilizando a linguagem Java e XML. Para o armazenamento dos dados, foi utilizado o banco de dados SQLite. Vale ressaltar que o TME é totalmente gratuito.

Ao iniciar o processo de interação com o TME e antes de o usuário obter acesso ao menu principal, é necessário inserir uma conta do Google para manter as estatísticas a serem exibidas ao usuário. Essa restrição se deve à facilidade em estabelecer este vínculo, tendo em vista que todo usuário do sistema operacional Android deve possuir uma conta Google para acesso a Play Store, local no qual o usuário realiza o download de aplicações. O aplicativo, quando iniciado, estabelece uma conexão com a conta Google automaticamente, caso o dispositivo esteja conectado à internet. $\mathrm{O}$ vínculo do aplicativo com a conta Google oferece algumas vantagens, por exemplo, a manutenção automática de um ranking localizado na Play Games, que pode ser acessado por duas modalidades: social e todos. No primeiro caso, o ranking é composto apenas por pessoas que estão na lista de contatos do usuário do aplicativo TME. No segundo, há um ranking geral composto por todos os usuários do aplicativo.

Outro aspecto a ser ressaltado é a possibilidade de "conquistas" serem atribuídas com base em determinados feitos que o usuário consiga realizar durante o uso do aplicativo. As conquistas acumulativas mantêm um registro de desempenho desde o primeiro uso do aplicativo e, à medida que o usuário progride, sua porcentagem aumenta até que a próxima conquista seja desbloqueada. As conquistas diretas não armazenam o progresso entre os usos, portanto, a cada novo uso o progresso do usuário é reiniciado. Para desbloquear esse tipo de conquista, o usuário deverá completá-la em apenas um uso. As conquistas têm a finalidade de estimular o uso do aplicativo por seus usuários, pois, a cada conquista desbloqueada, os jogadores são recompensados com pontos de experiência adicionados em seu perfil de conta Google.

O usuário pode selecionar o nível das questões que deseja responder, bem como ter acesso às suas estatísticas de respostas, a saber: quantidade de acertos e erros em cada nível (fácil, médio ou difícil) e números totais de tentativas. A Figura 1 ilustra telas de cada um dos diferentes níveis de questões em um smartphone.
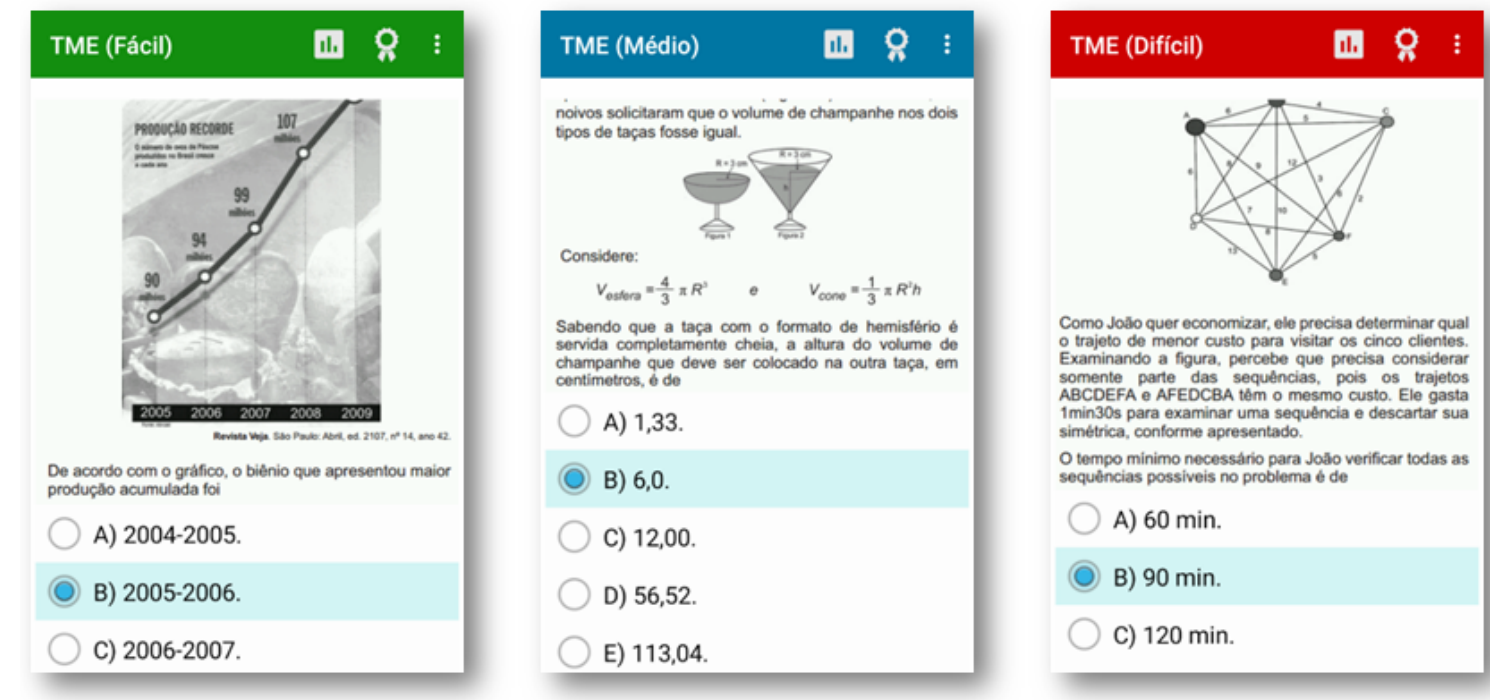

Figura 1- Exemplos de questões com base nos diferentes níveis. 
A interface foi projetada pata atuar de forma responsiva, visando melhor experiência do usuário independentemente do aparelho que estiver executando o aplicativo. Essa escolha se deve à grande variedade de dimensões das telas dos dispositivos atualmente, e principalmente em razão da diferença entre tablets e smartphones. Existe, ainda, a possibilidade de o usuário aplicar efeito de zoom no enunciado das questões, facilitando a leitura em dispositivos menores.

A cada questão respondida, uma mensagem notificando o acerto ou erro é exibida para o usuário. Em ambos os casos, a resolução passo a passo da respectiva questão é apresentada, fornecendo explicações adicionais para o esclarecimento de dúvidas. Essa decisão se deve ao fato de o ENEM ser caracterizado como um exame que testa não só os conhecimentos do estudante, mas sua resistência. Com isso, existe a possibilidade de o aluno apresentar dúvida e acabar optando pela resposta correta, mesmo sem ter o conhecimento requerido. Por esse motivo, mesmo em caso de acertos, a resolução da questão é exibida. A Figura 2 ilustra exemplos de mensagens de erro e acerto, o ranking dos usuários do aplicativo e estatísticas de respostas.
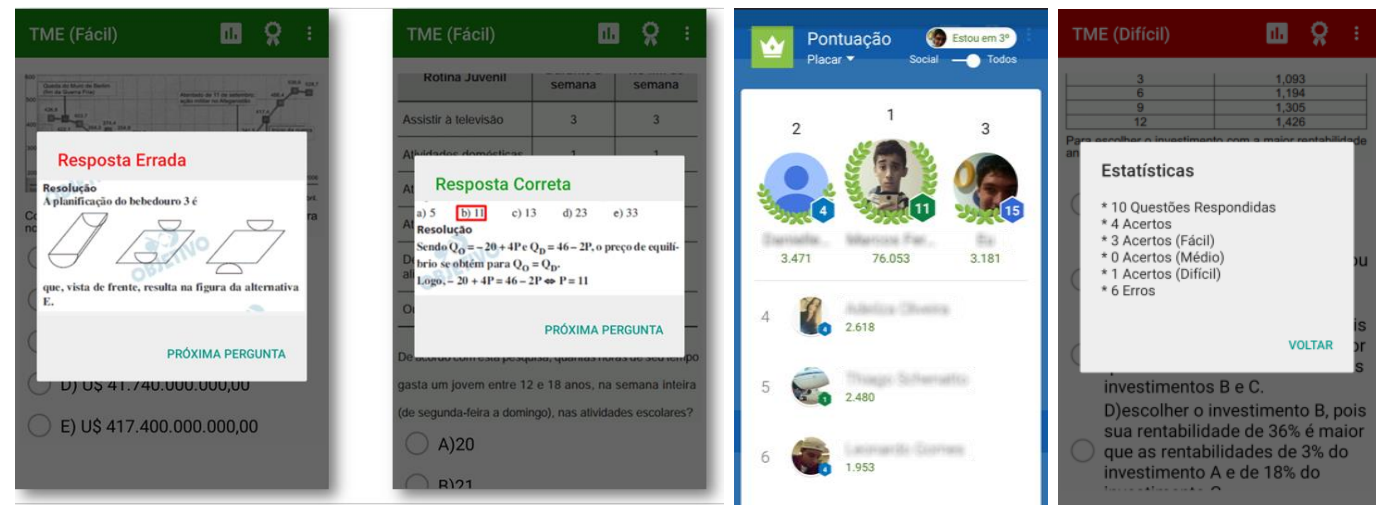

Figura 2- Exemplos de questões com base nos diferentes níveis.

\subsection{Participantes e Descrição do Método}

Os estudantes selecionados para participarem do estudo eram correspondentes ao $3^{\circ}$ ano do Ensino Médio e de um curso Pré-Vestibular (PV), com idade média de 18 anos. A pesquisa experimental foi realizada em uma instituição privada de ensino da cidade de Itajubá-MG. Inicialmente, foram selecionadas cinco turmas, que foram separadas em dois grupos: turma $\mathrm{A}$, que representa os alunos do $3^{\circ}$ ano, e turma $\mathrm{B}$, que corresponde aos alunos do PV. Os dois grupos juntos totalizaram 109 alunos presentes no primeiro encontro, que envolveu a apresentação do aplicativo. No entanto, somente parte deles utilizou de fato a aplicação no período disponível para experimentação e, por conseguinte, puderam participar da segunda etapa, que consistiu em responder um questionário avaliativo sobre o uso do aplicativo TME. A turma A foi composta por 15 homens e nove mulheres; a turma B por quatro homens e três mulheres, totalizando 31 estudantes participantes da pesquisa experimental.

O método para a aplicação da pesquisa experimental consistiu de duas etapas. Durante a primeira etapa, foram realizadas duas apresentações sobre a aplicação desenvolvida para os estudantes de ambas as turmas (A e B), com duração aproximada de 10 minutos cada. Para a realização da apresentação, foi preparado um breve material que foi exposto aos alunos com o intuito de demonstrar o aplicativo. Após o término das apresentações, os estudantes foram convidados a utilizar o aplicativo disponível gratuitamente na Play Store. A segunda etapa foi realizada um mês após a primeira. Esse 
V Congresso Brasileiro de Informática na Educação (CBIE 2016)

Anais do XXVII Simpósio Brasileiro de Informática na Educação (SBIE 2016)

intervalo de tempo foi necessário para que os alunos pudessem utilizar o aplicativo.

Após este período, foi realizada a aplicação de um questionário avaliativo sobre o uso experimentado do aplicativo pelos estudantes. O questionário consistiu em 10 questões de múltipla escolha, cujo objetivo foi minimizar a subjetividade de questões abertas, além de oferecer um espaço para a descrição de aspectos relevantes caso os alunos desejassem manifestar comentários adicionais. Durante a aplicação do questionário, os autores da pesquisa não interferiram ou ajudaram os participantes em respondê-lo. Os participantes foram informados sobre o objetivo da atividade a qual foram submetidos, bem como sobre o anonimato e uso dos dados coletados apenas para fins de pesquisa. O Quadro 1 apresenta as questões investigadas, além de uma questão final que solicita comentários de ordem geral.

\section{Questões}

Q1. Com que frequência semanal você fez uso do aplicativo?

Q2. De maneira geral, a utilização do aplicativo foi de fácil compreensão e assimilação?

Q3. Os recursos visuais disponibilizados pelo aplicativo (cores, ícones e imagens) são de boa qualidade?

Q4. Você acha que o uso do aplicativo contribuiu para um treinamento adequado para o ENEM?

Q5. Você acha que o uso do aplicativo contribuiu para auxiliar a aprendizagem de matemática?

Q6. Você gostaria que o aplicativo tivesse suporte a outras disciplinas, tais como português, inglês, redação, história, geografia etc.?

Q7. De maneira geral, como você avalia a performance do aplicativo no seu smartphone?

Q8. A qualidade e a quantidade de questões disponíveis no aplicativo foram adequadas?

Q9. O espaço necessário para o armazenamento do aplicativo no smartphone foi adequado?

Q10. O aplicativo é de fácil instalação e desinstalação?

Comentários adicionais:

Quadro 1 - Questões da avaliação qualitativa sobre a utilização do TME.

\section{Análise dos Dados}

A primeira questão da avaliação buscou identificar a frequência de uso do aplicativo durante o intervalo entre as duas etapas. De modo geral, 62\% dos participantes utilizaram o TME pelo menos três vezes na semana. A participação do público masculino foi um pouco maior, com uso médio semanal aproximado de 3,5 vezes. No caso das mulheres, o uso semanal foi de 2,5 vezes, aproximadamente. Em relação às turmas, o uso médio foi diferente. A turma A ( $3^{\circ}$ ano) realizou um uso mais frequente, alcançando uma média de 3,7 vezes na semana. Já a turma $B$, utilizou apenas 1,5 vezes na semana.

A segunda questão está relacionada à usabilidade do aplicativo. Nesse contexto, $100 \%$ (71\% atribuíram totalmente e $29 \%$ responderam muito) dos participantes afirmaram que a ferramenta apresenta boa facilidade de uso. Esse resultado é ilustrado na Figura 2. Considerando os gêneros, $84,6 \%$ do público feminino avaliou a usabilidade como totalmente adequada ( $15,4 \%$ responderam muito), enquanto que, para os homens, este índice foi de $63,2 \%$ (36,8\% responderam muito). Considerando cada turma, ambas 
V Congresso Brasileiro de Informática na Educação (CBIE 2016)

Anais do XXVII Simpósio Brasileiro de Informática na Educação (SBIE 2016)

apontaram $100 \%$ de aprovação, sendo esta porcentagem composta por $70,8 \%$ dos alunos que estavam totalmente de acordo na turma $\mathrm{A}$. Na turma B, 57,1\% dos participantes manifestaram essa mesma opinião (totalmente).

A próxima investigação (questão 3) diz respeito ao aplicativo oferecer os recursos visuais necessários para uma boa interação segundo a visão dos estudantes. Neste caso, 97\% (68\% responderam excelente e $29 \%$ atribuíram bom) dos participantes concordaram que os recursos visuais são de boa qualidade. Somente $3 \%$ dos participantes apontaram essa resposta como regular. A aprovação do público feminino foi maior, pois $84,6 \%$ das mulheres relataram que os recursos são excelentes. Para os homens, este número corresponde a 57,9\% (36,8\% responderam bom). Para as turmas, foi obtida uma aceitação excelente de $62,5 \%$ por parte do $3^{\circ}$ ano $(37,5 \%$ responderam bom); já na turma do $\mathrm{PV}, 71,4 \%$ dos participantes manifestaram opinião excelente $(14,3 \%$ afirmaram bom $)$.

A quarta questão visou determinar se, de fato, o aplicativo cumpriu com seu propósito de auxiliar no estudo para o ENEM. Foi possível constatar que 93\% dos estudantes classificaram essa contribuição como totalmente $(48 \%)$ ou muito $(45 \%)$, enquanto apenas 7\% classificaram como regular. Essa opinião neutra pode ser atribuída ao fato de que alguns alunos apontaram a falta do monitoramento do tempo durante o uso do TME como um recurso essencial ao treinamento. De acordo com o sexo, a avaliação feminina foi $100 \%$ positiva ( $46,2 \%$ totalmente e $53,8 \%$ muito), enquanto $90 \%$ dos homens foram favoráveis (47,4\% totalmente e $42,1 \%$ muito). Analisando as turmas, os alunos do $3^{\circ}$ ano foram unânimes quanto à aprovação (50\% totalmente e $50 \%$ muito). Para a turma do PV, 71,4\% dos participantes aprovaram a contribuição, porém 28,5\% apontaram opinião regular nesta questão. Provavelmente, o fato de os alunos do PV terem usado o TME com menor regularidade (questão 1) tenha refletido nessa resposta.

Em relação à contribuição para o aprendizado de matemática, $75 \%$ dos estudantes manifestaram opinião favorável nesta questão, os demais afirmaram que a contribuição foi regular ou pouca. Esse índice pode ser justificado pelo fato de que o aplicativo não oferece materiais para ensinar o conteúdo de matemática, mas sim exercícios que visam treinar o conteúdo previamente estudado. Nesta questão, 73,7\% dos homens manifestaram opiniões positivas, enquanto que, para as mulheres, esta porcentagem foi de $77 \%$. Apesar de $83 \%$ da turma do $3^{\circ}$ ano ter opinado favoravelmente em relação a essa questão, 42,9\% dos estudantes da turma do PV se mostraram neutros, afirmando que a contribuição foi regular, e 14,3\% manifestaram pouco como resposta. Novamente, esses resultados podem ser justificados pelas respostas da questão 1.

A sexta questão foi elaborada com o objetivo de verificar a aceitação desse tipo de aplicação para o aprendizado. De acordo com os resultados, 100\% dos estudantes se mostraram favoráveis a esta iniciativa. Ademais, alguns participantes sugeriram que os autores deveriam expandir o aplicativo para outras disciplinas. Em relação aos gêneros e às turmas, a aprovação foi unânime.

A sétima questão - "De maneira geral, como você avalia a performance do aplicativo no seu smartphone?" - teve como objetivo avaliar se o aplicativo se comportou de maneira adequada nos mais diversos dispositivos. Neste caso, $90 \%$ dos estudantes manifestaram que o desempenho foi excelente (61\%) ou bom (29\%), enquanto os demais o classificaram como regular. As opiniões neutras podem ser explicadas devido à grande variedade de dispositivos disponíveis no mercado atualmente, 
V Congresso Brasileiro de Informática na Educação (CBIE 2016)

Anais do XXVII Simpósio Brasileiro de Informática na Educação (SBIE 2016)

com diferentes capacidades de processamento. Provavelmente, o desempenho tenha sido regular em determinados modelos.

No caso da oitava questão, $87 \%$ dos participantes afirmaram que o aplicativo está totalmente ou muito de acordo com a qualidade e a quantidade de questões presentes, enquanto $13 \%$ dos estudantes classificaram esses quesitos como regular ou pouco. De acordo com os sexos, $100 \%$ das mulheres estão totalmente ou muito de acordo. Para os homens, esta porcentagem alcançou 78,9\% das opiniões. Em relação às turmas, os estudantes do PV foram unânimes quanto à aprovação. A turma do $3^{\circ}$ ano foi favorável com $83,3 \%$ das respostas.

No que compete à questão $9,74 \%$ dos participantes ficaram satisfeitos com o tamanho de armazenamento do aplicativo. Dentre aqueles que manifestaram opinião negativa (apenas 3\%), este posicionamento pode ser explicado pela grande variedade de tamanho das memórias dos smartphones atualmente. Para os usuários que possuem certa limitação deste recurso, o armazenamento do aplicativo pode não ter sido adequado em função deste fato. Em relação aos sexos e às turmas, a situação foi semelhante.

A última questão investigada comprova a facilidade para instalação do aplicativo. O TME é compatível com diversas versões do Android, abrangendo qualquer versão acima da 2.3.3. A instalação se torna possível por meio da Play Store, na qual o aplicativo está disponível de forma gratuita para download. Neste âmbito, 100\% dos estudantes alegaram não haver dificuldades para instalar ou desinstalar o aplicativo. Este é um fator de suma importância, visto que a partir da facilidade de acesso ao aplicativo, sua utilização torna-se mais atrativa no contexto educacional, sobretudo se incorporando mais presente no dia a dia dos estudantes. Analisando os gêneros e as turmas, o resultado foi o mesmo.

\section{Considerações Finais}

Os aplicativos são ferramentas poderosas capazes de realizar as mais diversas tarefas. Contudo, recorrer a aplicativos pagos pode tornar seu uso oneroso e, desta forma, inviável. Os aplicativos gratuitos permitem democratizar o acesso ao conhecimento e à informação.

Com o avanço e o baixo custo de acesso à tecnologia, os smartphones se difundiram pela sociedade. Em virtude disso, centenas de aplicativos surgem diariamente. A categoria de aplicações educacionais está em constante expansão e, atualmente, existem diversos projetos de aplicativos educacionais gratuitos desenvolvidos para atender desde a educação infantil até a superior. Nesta pesquisa, foi concebido o TME, que é um aplicativo direcionado ao treinamento da disciplina de matemática para o ENEM.

Os resultados observados a partir da avaliação da ferramenta constatam que, de modo geral, houve aprovação dos estudantes com relação ao uso do TME. Os comentários relatados pelos participantes da pesquisa corroboram o resultado por meio de inúmeras opiniões positivas. A maioria dos estudantes alegou que o aplicativo proporcionou apoio adequado ao complementar o treinamento na disciplina de matemática para o ENEM. Além disso, as estatísticas geradas pelo Google Play apontam que o aplicativo atualmente conta com 68.796 downloads e que ainda está instalado em 14.773 smartphones.

Verifica-se, assim, que os aplicativos educacionais têm grande potencial para 
V Congresso Brasileiro de Informática na Educação (CBIE 2016)

Anais do XXVII Simpósio Brasileiro de Informática na Educação (SBIE 2016)

complementar o processo de aprendizagem. Porém, a falta de iniciativas na criação de aplicativos desta natureza e a mudança cultural de muitos docentes ainda são os principais desafios encontrados com relação a este tema.

\section{Referências}

Batista, S. C. F.; Behar, P. A.; Passerino, L. M. (2010). Recursos pedagógicos para dispositivos móveis: uma análise com foco na matemática. RENOTE: Revista Novas Tecnologias na Educação, v. 8, n. 3.

Batista, S. C. F.; Behar, P. A.; Passerino, L. M. (2011). M-learnMat: aplicação de um modelo pedagógico para atividades de m-learning em matemática. Anais do XXII Simpósio Brasileiro de Informática na Educação - SBIE, Aracaju.

Carvalho, D. L. (2009). Metodologia do ensino da matemática. 3. Ed. São Paulo: Cortez Editora.

Castro, M. H. G.; Tiezzi, S. (2004). A reforma do ensino médio e a implantação do ENEM no Brasil. Desafios, v. 65, n. 11, p. 46-115.

Cazorla, I. M. (2012). Metodologia do ensino da matemática. 3. Ed. Bahia: Editus Editora.

Lopes, A. C.; López, S. B. (2010). A performatividade nas políticas de currículo: o caso do ENEM. Educação em revista, v. 26, n. 1, p. 89-110.

Marçal, E. et al. (2010). Da elicitação de requisitos ao desenvolvimento de aplicações de mobile learning em matemática. Anais do XXI Simpósio Brasileiro de Informática na Educação - SBIE, João Pessoa.

Marçal, E.; Andrade, R.; Rios, R. (2005). Aprendizagem utilizando dispositivos móveis com sistemas de realidade virtual. RENOTE: Revista Novas Tecnologias na Educação, v. 3, n. 1.

McGreal, R. (2005). Mobile devices and the future of free education. Proceedings of ICDE World Conference, International Council for Distance Education.

MEC. Ministério da Educação. (2015). Portal do MEC: Legislação. Brasília. Disponível em: < http://portal.mec.gov.br/index.php $>$

Neto, J. F. B.; Fonseca, F. S. (2013). Jogos educativos em dispositivos móveis como auxílio ao ensino da matemática. RENOTE: Revista Novas Tecnologias na Educação, v. 11, n. 1 .

Nyiri, K. Towards a philosophy of m-learning. (2002). Proceedings of IEEE International Workshop on Wireless and Mobile Technologies in Education WMTE.

Ponte, J. P. et al. (2007). Programa de matemática do ensino básico. Ministério da Educação.

Ponte, J. P.; Souza, H. (2010). Uma oportunidade de mudança na matemática no ensino básico. Lisboa: APM.

Rheingold, H. (2007). Smart mobs: the next social revolution. Basic Books.

Sena, D. M.; Oliveira, E. H.; Carvalho, L. S. (2014). Aplicativos móveis para o aprendizado de matemática. Anais do XXV Simpósio Brasileiro de Informática na Educação - SBIE, Dourados.

Silva, E. F.; Ribas, M. H. (2009). A prova do ENEM: o que pensam os professores de matemática? Olhar de Professor, v. 6, n. 1. 